\title{
Saúde sexual feminina em tempos de empoderamento da mulher
}

\author{
Female sexual health in women's empowerment times \\ La salud sexual femenina en los tiempos de empoderamiento de las mujeres
}

Recebido: 23/05/2021 | Revisado: 30/05/2021 | Aceito: 07/06/2021 | Publicado: 21/06/2021

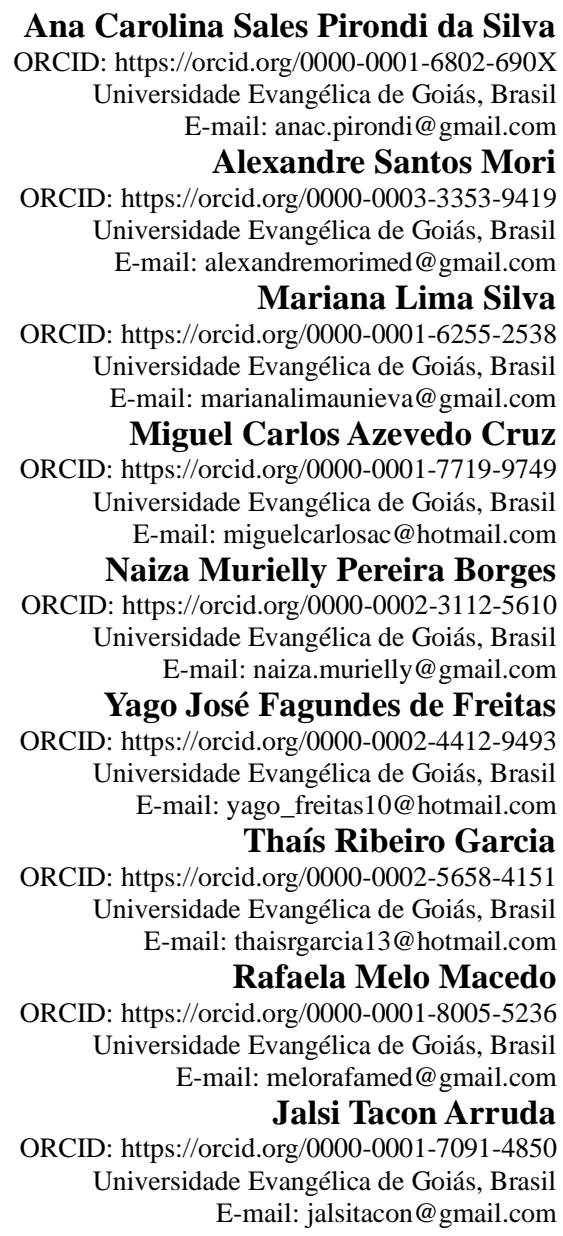

\section{Resumo}

O conceito de sexualidade tem se modificado ao longo dos anos. É um dos indicadores de qualidade de vida, parte integrante da saúde e do bem-estar geral, não se restringindo a meta reprodutiva apenas, fortalece relações afetivas entre pessoas. A sexualidade feminina tem se alterado de modo que hoje se discute mais sobre questões antes impensáveis. No entanto, ainda há lacunas no conhecimento que devem ser estudadas para quebrar paradigmas e desfazer conceitos errôneos. O presente estudo realizou uma revisão integrativa da literatura sobre a saúde sexual feminina. Estudos originais foram obtidos nas bases de dados PubMed e SciELO utilizando os descritores: disfunção sexual, saúde sexual feminina, saúde da mulher, em português e inglês. O tema é extremamente amplo abrangendo questões físicas, anatômicas, sociais, biológicas, psicoemocionais além de diversos fatores externos. A vida sexual da mulher impacta diretamente na sua qualidade de vida e por ser complexa e individualizada, carece da atuação conjunta das diversas áreas do conhecimento e da saúde.

Palavras-chave: Direitos humanos; Disfunção sexual; Sexualidade.

\section{Abstract}

The concept of sexuality has changed over the years. It is one of the indicators of quality of life, an integral part of health and general well-being, not being restricted to the reproductive goal only, it strengthens affective relationships between people. Female sexuality has changed so that today we discuss more about previously unthinkable issues. However, there are still gaps in knowledge that must be studied to break paradigms and undo misconceptions. The 
present study carried out an integrative review of the literature on female sexual health. Original studies were obtained from the PubMed and SciELO databases using the descriptors: sexual dysfunction, female sexual health, women's health, in Portuguese and English. The theme is extremely broad, covering physical, anatomical, social, biological, psycho-emotional issues as well as several external factors. The sexual life of women has a direct impact on their quality of life and because it is complex and individualized, it lacks the joint action of the various areas of knowledge and health.

Keywords: Human rights; Sexual dysfunction; Sexuality.

\section{Resumen}

El concepto de sexualidad ha cambiado a lo largo de los años. Es uno de los indicadores de calidad de vida, parte integral de la salud y el bienestar general, no restringiéndose únicamente al objetivo reproductivo, fortalece las relaciones afectivas entre las personas. La sexualidad femenina ha cambiado por lo que hoy hablamos más sobre temas impensables antes. Sin embargo, todavía existen lagunas en el conocimiento que deben estudiarse para romper paradigmas y deshacer conceptos erróneos. El presente estudio realizó una revisión integradora de la literatura sobre salud sexual femenina. Los estudios originales se obtuvieron de las bases de datos PubMed y SciELO utilizando los descriptores: disfunción sexual, salud sexual femenina, salud de la mujer, en portugués e inglés. El tema es extremadamente amplio y abarca cuestiones físicas, anatómicas, sociales, biológicas, psicoemocionales y varios factores externos. La vida sexual de las mujeres tiene un impacto directo en su calidad de vida y por ser compleja e individualizada carece de la acción conjunta de las distintas áreas del conocimiento y la salud.

Palabras clave: Derechos humanos; Disfunción sexual; Sexualidad.

\section{Introdução}

A sexualidade é uma das dimensões do ser humano que envolve gênero, identidade e orientação sexual, erotismo, envolvimento emocional, amor e reprodução (Abdo, 2010; Mendonça et al., 2017). Envolve além do corpo, história, costumes, relações afetivas e culturais (Diamond \& Alley, 2019). Hoje, o sexo faz parte do cotidiano das pessoas não estando limitado apenas a concepção, já que o prazer do ser humano independe da reprodução, extrapolando, também, os aspectos orgânicos e associando-se a estes os fatores biopsicossociais (Ventriglio \& Bhugra, 2019; Cavalcante et al., 2021).

O conceito de sexualidade é algo fluido que tem se modificado ao longo dos anos (Lamont et al., 2017). A Organização Mundial de Saúde define que a sexualidade é um aspecto central do ser humano que é vivido e expresso de diversas formas como em pensamentos, fantasias, desejos, crenças, atitudes, valores, comportamentos, práticas, papéis e relacionamentos e é influenciada pela interação de fatores biológicos, psicológicos, sociais, econômicos, políticos, culturais, legais, históricos, religiosos e espirituais (Correa et al., 2015; Campos et al., 2016; Barreto et al., 2018; Kingsberg et al., 2019; Cortes et al., 2021).

A sexualidade é também um dos indicadores de qualidade de vida, sendo, portanto, parte integrante da saúde e do bem-estar geral, não se restringindo a meta reprodutiva apenas, mas também fortalece relações amorosas e afetivas entre as pessoas (Antonio et al., 2016; Diamond \& Alley, 2019). Desse modo, percebe-se a importância da saúde sexual e sua relação intrínseca com os diversos domínios que compõem a qualidade de vida (Campos et al., 2016; Batista et al., 2017; Rojas Cabrera et al., 2017).

A saúde sexual feminina é definida como a habilidade da mulher desfrutar e expressar sua sexualidade, de forma positiva, informada e segura, sem risco de doenças sexualmente transmissíveis, gravidez indesejada e coerção (Correa et al., 2015; Campos et al., 2016; Kingsberg et al., 2019). Na sociedade, onde é marcante a desigualdade de gênero, as relações de poder e frequentes ameaças de violência por vezes impossibilitam a mulher de realizar práticas sexuais seguras, ressaltando-se, por exemplo, o baixo poder de decisão na escolha e uso de um método contraceptivo (Barbosa et al., 2015; Bezerra et al., 2016; Baigorria et al., 2017; Pinto et al., 2017; Delziovo et al., 2018; Gaspar \& Pereira, 2018; Sousa \& Silva, 2018; Oliveira et al., 2019; Mafioletti et al., 2020; Duffrayer et al., 2021).

A disfunção sexual pode afetar tanto homens quanto mulheres (Ribeiro \& Valle, 2016). Em relação à disfunção sexual feminina (DSF), esse distúrbio está diretamente ligado a falta de desejo sexual da mulher, dor durante a relação sexual, 
dificuldade em ficar excitada, incapacidade de atingir o orgasmo ou lubrificação insuficiente. A resposta sexual feminina, diferentemente da masculina, se divide em 4 fases: desejo, excitação, orgasmo e resolução. Uma perturbação em um destes estágios ou ainda a presença de dor associada ao ato sexual caracteriza a disfunção sexual (Abdo, 2010; Batista et al., 2017). No caso feminino a disfunção sexual é caracterizada por uma perturbação clinicamente significativa na capacidade da mulher responder ou experimentar prazer sexual (Ribeiro \& Valle, 2016; Barreto et al., 2018).

As disfunções sexuais femininas são um problema multifatorial, envolvendo determinantes anatômicos, vasculares, neurológicos e hormonais, além de psicológicos e interpessoais. Além disso, geram significativo impacto emocional que pode agravar o quadro e tem influência negativa em diversos aspectos da vida da mulher (Antonio et al., 2016; Barreto et al., 2018). Com os avanços da medicina, educação e tecnologia, aumentou-se a expectativa de vida da população de modo que a sexualidade saudável se tornou um aspecto importante também em idades mais avançadas (Nishioka, 2018; Ventriglio \& Bhugra; 2019). Tal fato traz novos desafios, visto que com a longevidade ocorre uma diminuição na produção dos hormônios sexuais, ocasionando uma redução qualitativa e quantitativa da resposta sexual, que se acentua com o avançar da idade.

A sexualidade feminina tem se alterado ao longo do tempo, de modo que hoje se discute mais sobre questões antes impensáveis (Ventriglio \& Bhugra, 2019). No entanto, ainda há lacunas no conhecimento e muito deve ser estudado por novas pesquisas na área para continuar quebrando paradigmas e desfazendo conceitos errôneos (Diamond \& Alley, 2019). Dessa forma, o presente estudo realizou uma revisão integrativa da literatura para atualização dos conhecimentos sobre a saúde sexual feminina.

\section{Metodologia}

Trata-se de uma revisão integrativa da literatura que tem por finalidade sintetizar resultados obtidos em pesquisas, de maneira ordenada e abrangente, integrando o conhecimento sobre um determinado tema. A revisão integrativa foi realizada a partir de buscas por estudos originais utilizando as bases de dados PubMed (United States National Library of Medicine) e SciELO (Scientific Eletronic Library Online). Foram utilizados os seguintes Descritores em Ciências da Saúde, que constam na plataforma DeCS da Biblioteca Virtual de Saúde (BVS): disfunção sexual, saúde sexual feminina, saúde da mulher, que foram utilizados em combinação, com auxílio de operadores Booleanos (and/or), em português e inglês. A coleta de dados foi realizada entre março de 2020 a março de 2021.

Os critérios de inclusão foram: artigos originais ou de revisão completos que investigaram a saúde sexual feminina; publicados no período de 2010 a 2021; sem restrição de idioma de publicação. Os estudos incluídos na revisão integrativa permitem uma avaliação crítica do tema discutido sendo possível identificar lacunas que poderão direcionar futuras pesquisas. Foram excluídos estudos que não investigassem o tema proposto, artigos de opinião, carta ao editor, e que não atendessem aos critérios de inclusão descritos. A primeira etapa foi identificar os estudos e se preenchiam os critérios estabelecidos, passando por uma seleção inicial das publicações realizada com leitura do título e resumo disponível para verificar se enquadravam na temática. Após essa etapa, os estudos previamente selecionados foram lidos na íntegra, empregando os critérios de inclusão e exclusão, para análise e obtenção das informações necessárias para a construção do presente estudo. Caso houvesse discordância entre os avaliadores sobre os critérios analisados, foi realizada discussão específica sobre o artigo em questão, confrontando ideias com base nos critérios adotados para a revisão.

\section{Resultados e Discussão}

A sexualidade é uma condição inerente a identidade humana, se desenvolve ao longo da vida (Diamond \& Alley, 2019). É um fator intrínseco a qualidade de vida, que leva a diferentes formas de buscas, compreensão e vivências do prazer. 
Apresenta um caráter social e histórico com particularidades em cada espaço e tempo, sendo, portanto, um elemento fundamental da condição de ser humano (Moraes \& Vitalle, 2012).

Em relação à saúde sexual, seu conceito é amplo e abrangente (Lamont et al., 2017; Kingsberg et al., 2019). Inclui desde um desenvolvimento sexual saudável, relacionamentos responsáveis com prazer sexual, até ausência de enfermidades, deficiências, violência e práticas que envolvam risco sexual (Abdo, 2010; Dantas et al., 2014; Sousa et al., 2015; Campos et al., 2016; Baigorria et al., 2017; Gaspar \& Pereira, 2018). É a habilidade de expressar a sexualidade, possibilitando experimentar uma vida sexual agradável e segura (Pinto et al., 2017; Diamond \& Alley, 2019). Valoriza, portanto, as relações pessoais, a expressão da identidade e a própria vida, sendo enriquecedora e estimuladora (Correa et al., 2015; Bezerra et al., 2016; Delziovo et al., 2018).

A saúde sexual feminina tem tido avanços, fato inegável. Um dos pontos que merece ser citado é a crescente melhoria do acesso aos serviços de saúde, com destaque para a Atenção Básica (Campos et al., 2016; Lamont et al., 2017). Têm-se percebido maiores taxas de diagnóstico e tratamento das Infecções Sexualmente Transmissíveis (IST), além de estratégias de prevenção (Correa et al., 2015). Aumento no acompanhamento pré-natal e o planejamento familiar, realização de exames preventivos, distribuição gratuita de preservativos, dentre outros procedimentos de promoção da saúde.

Há, porém, empecilhos fortes neste processo. A busca da Atenção Básica pela integralidade da saúde sexual feminina ainda conta com a presença da polarização "mulher x reprodução" e "homem x sexualidade" na mentalidade social (Oliveira et al., 2018; Kingsberg et al., 2019). Também não são todas as mulheres que buscam o serviço de saúde (Correa et al., 2015). Das que buscam, quando constatado que necessitam de tratamento, 6\% não conseguem o acesso. E quanto as notificações, infelizmente as taxas ainda são baixas em comparação com a totalidade dos casos.

Ainda no contexto da saúde, organizações e sociedades científicas vêm incluindo temas relacionados à sexualidade humana, de modo geral. O desenvolvimento de Guias ou Guidelines sobre o tema vêm ganhando espaço e trabalhos sobre saúde sexual da mulher têm aumentado (Lamont et al., 2017; Kingsberg et al., 2019). Na prática, porém, ainda há um longo caminho a ser percorrido. Mesmo os especialistas não investigam de forma regular a saúde sexual das pacientes, por constrangimento ou falta de tempo. E, se o médico não pergunta, a paciente muitas vezes acredita que o assunto não é relevante na consulta (Diamond \& Alley, 2019).

Sobre as IST, tem ocorrido um avanço perceptível na prevenção, rastreio, diagnóstico e tratamento. No entanto, notase que nem todas as pacientes procuram atendimento, não se considerarem vulneráveis acreditando que há apenas um grupo específico de risco para IST. Há, portanto, neste e em demais temas relacionados, muitas dúvidas e incertezas sobre a saúde sexual.

Há uma crescente consolidação e o aprimoramento de medidas de apoio voltadas para assegurar os direitos constitucionais à mulher, de liberdade e sexualidade no Brasil (Moraes \& Vitalle, 2012; Viana \& Sousa, 2014). Dois exemplos são a Secretaria Nacional de Políticas para as Mulheres, criada em 2003 e a Lei n ${ }^{\circ}$ 10.778/2003, que estabelece a notificação compulsória de casos de violência contra a mulher atendida em serviços de saúde públicos ou privados (Barbosa et al., 2015; Pinto et al., 2017; Duffrayer et al., 2021). Essa lei foi alterada pela Lei no 13.931/2019 que dispõe sobre a notificação compulsória dos casos de suspeita de violência contra a mulher (Bezerra et al., 2016; Pinto et al., 2017; Sousa \& Silva, 2018; Delziovo et al., 2018; Oliveira et al., 2019).

Entretanto, muitas das vezes não é vista a aplicação prática de tais medidas. As taxas de não notificação, no caso da violência sexual, por exemplo, ainda superam 20\%, onde os motivos apontados para tal situação são desinformação e despreparo dos profissionais, além da dificuldade para preenchimento da ficha em si (Sousa et al., 2015; Baigorria et al., 2017; Sousa \& Silva, 2018). Também se nota descumprimento dos protocolos e fluxogramas existentes, bem como a falta de uma 
rede estruturada de atenção na qual a Atenção Primária seja o local de prioridade de atendimento (Gaspar \& Pereira, 2018; Oliveira et al., 2019; Duffrayer et al., 2021).

É importante destacar que a percepção de satisfação sexual feminina é diferente da masculina. Um estudo mostrou que, para as mulheres, a satisfação sexual está muito ligada ao contexto emocional e a qualidade de seus encontros sexuais, o que para os homens eles valorizam principalmente a frequência (Scott et al., 2018).

Sobre os diferentes pontos de vista, há a relevância de um passado histórico que se repercute ainda no presente. A tradição patriarcal na qual por muito tempo o marido era tido como o provedor da casa e a esposa, na situação de dependente, era encarregada dos "deveres conjugais", incluindo, nestes o "serviço sexual" (Dantas-Berger \& Giffin, 2005; Diamond \& Alley, 2019). Felizmente tem ocorrido mudanças nesse modelo, com a crescente participação das mulheres no mercado de trabalho e conquista de seu espaço na sociedade (Viana \& Sousa, 2014). Daí o seguinte questionamento: o que tais mudanças significam, de verdade, no âmbito da sexualidade feminina?

Pode-se responder a esse questionamento dizendo que, mesmo com toda esta mobilização em prol da autonomia feminina e da mudança do estilo de vida das mulheres desde o século passado, sua representatividade sexual tem se mantido estigmatizada (Ventriglio \& Bhugra; 2019). Por vezes, ainda continuam sendo vistas como sujeitas a ordem masculina, sendo que algumas dessas próprias mulheres têm interiorizadas tais concepções. Ou não têm conhecimento sobre sua própria sexualidade, principalmente sobre o orgasmo, o que contribui para aceitação de um cenário no qual, por vezes, não é satisfatório para si mesmas (Viana \& Sousa, 2014; Ribeiro \& Valle, 2016).

Com essa visão, a Organização das Nações Unidas estabeleceu como o terceiro dos oito Objetivos do Milênio a autonomia feminina, bem como a igualdade de gênero. Portanto, seja em uma relação heterossexual ou não, é fundamental a mulher conhecer a si própria e entender os fatores que interferem em sua saúde sexual (Ventriglio \& Bhugra; 2019). A sexualidade não diz respeito só a componentes naturais, mas também a fatores inconscientes, culturais e a expressão do próprio corpo, ou seja, tem caráter fortemente subjetivo (Dantas et al., 2014; Sousa \& Silva, 2018; Diamond \& Alley, 2019).

Neste processo, merecem enfoque dois conceitos: empoderamento e autoadvocacia. O primeiro engloba a ideia de tornar-se capaz de fazer escolhas de acordo com seus desejos e responsabilizar-se por suas decisões. Aliado a ele, a autoadvocacia traz o princípio de que a pessoa tem o direito e o dever de participar de decisões sobre si própria (Moraes \& Vitalle, 2012; Dantas et al., 2014; Viana \& Sousa, 2014).

Ambos os conceitos levam ao protagonismo feminino em relação ao autoconhecimento e a redução da condição de passividade. Isso auxilia as mulheres na desconstrução do estigma feminino do passado, e torna possível compreender a complexidade de sua sexualidade (Ventriglio \& Bhugra; 2019). Quando se entende a importância de se saber separar propriamente o prazer sexual da função reprodutiva, a qualidade da saúde sexual se eleva. E, por conseguinte, a própria capacidade reprodutiva também melhora (Dantas et al., 2014; Diamond \& Alley, 2019).

Um meio importante de se alcançar este entendimento é através do ensino nas escolas. Um estudo japonês avaliou o impacto de se incluir saúde sexual no ensino, acerca de sexualidade propriamente dita, planejamento familiar e prevenção de doenças sexualmente transmissíveis e notou-se que a falta de conhecimento das mulheres é um empecilho para que elas saibam tomar decisões (Nishioka, 2018). Portanto, é preciso compreender para poder buscar a própria saúde sexual.

Percebe-se, porém, que, quando presentes, tais discursos têm como foco os problemas associados a sexualidade, como gravidez indesejada por exemplo. Negligenciam explicações básicas, mas imprescindíveis, como sobre a própria sexualidade e sobre experiências sexuais serem normais e saudáveis. A sexualidade está presente desde o nascimento, porém sua expressão é desenvolvida por meio de um processo ao longo da vida, no qual orientações de qualidade são essenciais (Lamont et al., 2017).

A saúde sexual está ligada também à saúde mental (Fortes et al., 2021). A causa de um bloqueio sexual pode estar 
ligada a vivências traumáticas e repressoras, funcionando como um bloqueio externo (Oliveira et al., 2018). Há uma associação de transtornos psicológicos as seguintes categorias de distúrbios sexuais: desejo sexual hipoativo, aversão sexual, distúrbios de excitação sexual, anorgasmia e distúrbios durante o ato (dispareunia, vaginismo e dor sexual não coital).

A questão comportamental e orientação sexual merece destaque. A sexualidade moderna trouxe o conceito de fluidez sexual e de identidade de gênero para explicar como o gênero e a orientação sexual podem ser flexíveis. Por motivos religiosos, crenças políticas, dentre outros, há ainda discriminação e censura dos que não se enquadram no conceito de heterossexual (Cortes et al., 2021). É frequente até que variações sexuais sejam tidas como patológicas, que carecem de tratamento, o que acarreta graves danos psicológicos a estas mulheres (Ventriglio \& Bhugra; 2019).

No contexto de saúde sexual, é necessário também apontar que existem variações do que é considerado normal nos padrões da sexualidade e doenças que, estas sim, carecem de diagnóstico e tratamento. É uma questão complexa, mas considera-se doença se é um comportamento que afeta a liberdade de outros, se causa danos (IST por exemplo), se é socialmente inaceitável (exibicionismo, incesto, dentre outros) ou se é proibido por lei como o estupro (Ventriglio \& Bhugra; 2019).

As pressões sociais influenciam diretamente na política, logo, no que será aceitável perante a lei e o que será tido como ilegal. E, neste aspecto, profissionais da saúde, políticos e a população em geral devem conhecer e compreender tais definições, de forma a evitar a discriminação e constrangimentos desnecessários, que acarretam traumas e resultam em disfunções sexuais (Kingsberg et al., 2019; Ventriglio \& Bhugra, 2019).

Uma vez que a saúde sexual feminina tem caráter fortemente contextual, e devido a sua relação com a saúde mental, recomenda-se a avaliação de uma possível disfunção sexual a partir de três fatores (Oliveira et al., 2018; Fortes et al., 2021). O primeiro engloba os fatores predisponentes, ou seja, do passado da mulher, que possam ter refletido em seu desenvolvimento psicossocial, como um abuso sexual. O segundo abrange fatores que precipitam ou podem perpetuar a disfunção, no contexto atual da vida da mulher, sejam ambientais, interpessoais, dentre outros. E o terceiro fator diz respeito a ocorrências cirúrgicas ou médicas vividas pela mulher (Abdo, 2010; Correa et al., 2015; Antonio et al., 2016; Barreto et al., 2018).

Percebe-se que mesmo com tantos avanços para se alcançar o acesso e a totalidade da saúde sexual e sexualidade feminina ainda há uma série de desafios existentes. A partir da observação dos fatores supracitados, cabe salientar a extrema importância dos profissionais de saúde neste cenário. É incumbência deles conseguir orientar as mulheres sobre saúde sexual. Por ser uma questão rica e complexa, carece do envolvimento de profissionais de diversas áreas (ginecologia, urologia, psiquiatria, medicina de família etc.), para uma abordagem integral da sexualidade (Diamond \& Alley, 2019).

E, para isto, é necessário saber abordar a sexualidade com linguagem apropriada, sem coerção e sem causar traumas (Ventriglio \& Bhugra; 2019). Deve-se conseguir criar um ambiente acolhedor, onde a mulher possa estabelecer relação de confiança. O profissional deve estar aberto a discutir preocupações e dúvidas de caráter sexual, sendo sensível ao estágio de vida e a situação individual da mulher, havendo respeito pelos padrões de comportamento e orientação sexual de cada uma (Lamont et al., 2017).

No contexto da saúde sexual feminina a incidência exata da DSF ainda é incerta. Um estudo sobre o comportamento sexual realizado nos Estados Unidos, com cerca de 2000 mulheres de 18 a 59 anos, estimou uma prevalência de $43 \%$ de mulheres com disfunção, sendo a redução do desejo sexual a alteração mais comum identificada, em 22\% delas. No Brasil, um estudo similar realizado em sete estados, demonstrou uma prevalência de $49 \%$ de disfunção sexual feminina, sendo a diminuição do desejo sexual novamente a mais observada, em 26,7\% das mulheres (Antonio et al., 2016; Barreto et al., 2018).

A alta prevalência de tais disfunções tem se tornado cada vez mais evidente à medida que tem se realizado mais estudos sobre o assunto. O Estudo do Comportamento Sexual no Brasil, evidenciou que 49\% das 1.219 mulheres analisadas 
reportaram pelo menos um tipo de disfunção sexual, sendo a mais comum o transtorno do interesse sexual (Antonio et al., 2016). Outro estudo demonstrou uma epidemiologia semelhante estimando $40 \%$ a $45 \%$ das mulheres com algum grau de disfunção sexual (Barreto et al., 2018).

A DSF está muito relacionada ao desconforto pessoal e a má qualidade de vida. Possui relação direta com desgaste emocional por insatisfação sexual, principalmente entre as mulheres mais jovens (Antonio et al., 2016). Esta insatisfação abrange um grande percentual de mulheres e se associa a fatores biopsicossociais, onde, por muito tempo na sociedade, a importância do prazer feminino na relação sexual foi menosprezada, como já citado. E ao afetar a saúde mental resulta em baixa autoestima e até questionamentos acerca de sua sexualidade (Oliveira et al., 2018; Fortes et al., 2021).

O impacto emocional relacionado a DSF pode agravar o problema. A curto prazo, podem provocar transtornos emocionais os quais afetam vários aspectos da vida da portadora da DSF, como a família ou o trabalho. E, o interesse e a satisfação sexual se associam a problemas de expressividade emocional, a baixa autoestima, sentimentos de depressão e solidão e até mesmo a função cognitiva. Sobre tais categorias, estudos epidemiológicos realizados nos EUA, Suécia e Reino Unido concluíram que cerca de $40 \%$ das mulheres em idade reprodutiva apresentam queixas sexuais significativas, sendo: $33 \%$ delas com déficit no desejo sexual, 24\% com anorgasmia, 19\% com dificuldade de excitação e lubrificação, 15\% com dispareunia e 9\% com outras queixas (Antonio et al., 2016; Oliveira et al., 2018).

Além disso, nota-se que a prevalência e intensidade destes transtornos sexuais são superiores aos dos distúrbios sexuais masculinos. Porém, cabe ressaltar que os problemas femininos recebem menor atenção em relação aos masculinos, tanto sobre a quantidade de estudos dos aspectos fisiológicos e psicológicos da disfunção sexual, quanto na disponibilização de métodos terapêuticos (Batista et al., 2017). Quanto a isto, há uma variedade de instrumentos para avaliação da DSF. Questionários, principalmente de autorresposta, são usados com excelência para estudos de avaliação da função sexual. A grande quantidade e diversidade de questionários disponíveis, porém, reflete ausência de consenso e inexistência de um método eficaz para avaliação completa da função sexual feminina (Mendonça et al., 2017).

Outra questão relevante que impacta diretamente na saúde e sexualidade da mulher é a violência sexual (Gaspar \& Pereira, 2018; Oliveira et al., 2019; Mafioletti et al., 2020). Seu conceito não se refere apenas à penetração do corpo com pênis ou outro objeto. Inclui o estupro, agressão, molestamento, assédio e incesto. Abrange qualquer tentativa de ato sexual, como o ato sexual propriamente dito, comentários ou insinuações sexuais não desejadas. Também atos de tráfico ou contra a sexualidade de uma pessoa usando coerção, sem depender da relação do agressor com a vítima e em qualquer contexto (Barbosa et al., 2015).

Quanto a isto, os dados são alarmantes. Segundo o relatório sobre violência promovido pela Organização Mundial da Saúde, $35 \%$ das mulheres no mundo relatam ter sofrido violência física e/ou sexual alguma vez na vida e estima-se a ocorrência de aproximadamente um caso a cada minuto (Sousa et al., 2015; Gaspar \& Pereira, 2018). Os dados do Brasil mostram que uma, em cada dez mulheres, tem uma ocorrência de violência sexual na vida (Bezerra et al., 2016; Delziovo et al., 2018).

No Brasil 11,9 \% dos casos de violência contra a mulher são do tipo sexual. O Anuário Brasileiro de Segurança Pública indica uma média de 125 casos de estupro por dia, segundo pesquisa feita em 2015 (Baigorria et al., 2017; Gaspar \& Pereira, 2018; Mafioletti et al., 2020). Isso resulta em lesões físicas e psicológicas que impactam na vida da mulher e nas relações sociais. E ainda se torna uma demanda nos serviços de saúde, trazendo custos financeiros e humanos (Barbosa et al., 2015; Oliveira et al., 2019).

Dentre estas consequências das violências observa-se: danos irreparáveis e permanentes ligados ao bem-estar físico e mental, questões sexuais, sociais, reprodutivas e emocionais das mulheres agredidas, com destaque também para gravidez e 
infecções sexualmente transmissíveis (IST), incluindo HIV/AIDS entre tantas outras (Sousa et al., 2015; Oliveira et al., 2018; Cavalcante et al., 2021). A violência sexual não apenas é uma grave violação do direito humano, mas também um empecilho ao desenvolvimento socioeconômico de uma região e um sério problema de saúde pública (Moraes \& Vitalle, 2012; Bezerra et al., 2016; Delziovo et al., 2018; Sousa, Silva, 2018).

Deve-se reforçar que a sexualidade e disfunções sexuais são fortemente relacionados a causas externas. Fatores que refletem negativamente na saúde sexual, como ansiedade, estresse, e baixa autoconfiança, frequentemente se associam a outros problemas (Ribeiro \& Valle, 2016; Barreto et al., 2018). Dentre eles, merece destaque, pelo alto risco potencial, o uso de drogas (lícitas e/ou ilícitas). No meio universitário, principalmente, os índices de consumo de drogas são alarmantes, sendo o álcool e o tabaco os mais comuns.

O consumo de drogas causa prejuízos em série, podendo ter impacto direto na saúde sexual ou agindo de forma indireta. Diretamente, pode-se tomar como um dos inúmeros exemplos que os universitários que fizeram uso de drogas lícitas e/ou ilícitas não utilizaram preservativo nas últimas relações sexuais. E indiretamente, as drogas podem levar a comportamentos de risco, problemas sociais, legais e da saúde, no geral, que também vão refletir na saúde sexual.

Um outro ponto também importante de ser abordado é a sexualidade em mulheres de idade avançada. Com o aumento da expectativa de vida e o envelhecimento da população há uma preocupação especial com essas mulheres. Primeiramente, é necessário reconhecer que ocorrem mudanças anatômicas da terceira idade, também alterações biológicas e psicossociais (Cunha et al., 2015). É essencial, porém, que a premissa de que os idosos não têm vida sexual seja desconstruída, lembrando-se que sexualidade não se restringe apenas a região genital, abrangendo também o lado afetivo, essencial ao ser humano.

Merece destaque o crescimento da porcentagem de idosos com ISTs. Com o desenvolvimento de reposição hormonal e outros meios de melhorar o desempenho sexual, a população idosa tem se tornado cada vez mais sexualmente ativa. No entanto, a prevenção de ISTs não vem acompanhando esta evolução, devido a tabus e preconceitos sociais, aliados à falta de campanhas e medidas preventivas e ao não reconhecimento dos idosos como população de risco para estas doenças.

A preocupação com a saúde sexual na velhice passou a receber atenção recentemente (Scott et al., 2018). Os profissionais da área da saúde ainda não têm como prática a abordagem deste ponto nas consultas, principalmente em se tratando das mulheres idosas. Com isso, agravos comuns nesta faixa etária como vaginismo, dispareunia e a própria prevenção de IST acaba não sendo feita, e a promoção da saúde, tão preconizada, acaba sendo prejudicada (Cunha et al., 2015).

Portanto, para a inclusão efetiva deste tema - saúde sexual feminina, na prática ainda precisa que os jovens, profissionais de saúde e familiares conversem e discutam sobre sexualidade, a aceitação da sexualidade como sendo natural e de manifestações amorosas, e o fim da infantilização dos idosos. Dessa forma será possível abrir caminho para o fim do constrangimento em abordar este tema, facilita-se o processo de conhecimento para desenvolvimento da sexualidade e o estabelecimento de relacionamentos (Scott et al., 2018).

\section{Conclusão}

O tema "Saúde Sexual Feminina" é extremamente amplo. Resulta de um conjunto que abrange questões físicas, anatômicas, sociais, biológicas, psicoemocionais e diversos fatores externos. É um tema que vem ganhando espaço na sociedade. Contudo, ainda enfrenta muitas barreiras, no âmbito de preconceitos, medos, constrangimentos e falta de conhecimento e conscientização da sociedade sobre o assunto.

A vida sexual da mulher impacta diretamente na sua qualidade de vida, sendo de extrema importância. Mas ainda é pouco discutida. É um tema que abrange desde a formação da mulher, sua infância, adolescência, vida adulta e velhice. E por ser de grande complexidade e individualizada, carece da atuação conjunta de diversas áreas do conhecimento e da saúde. 
O aumento dos estudos sobre esse assunto reflete a crescente conquista da mulher de sua representatividade social e do reconhecimento de sua sexualidade. Porém, o caminho a seguir ainda é longo. Devem ser realizados mais estudos nesta área, de modo a promover uma disseminação do conhecimento, auxiliando na quebra dos paradigmas de desinteresse social e preconceito quanto à saúde e o prazer sexual da mulher.

Espera-se que com os dados atualizados sobre saúde sexual feminina possam ser utilizados junto as ligas acadêmicas e ao diretório acadêmico, assim como passar as informações obtidas a estudantes de outros cursos da área de saúde que trabalham a saúde da mulher. Ainda se espera que esses conhecimentos sejam aplicados as próprias mulheres, estudantes, profissionais e a toda população feminina.

\section{Referências}

Abdo, C. H. N. (2010). Considerações a respeito do ciclo de resposta sexual da mulher: uma nova proposta de entendimento. Diagnóstico \& Tratamento, $15(2): 88-90$.

Antonio, J. Z., Silva, A., Costa, P. P. B., Jung, D., Pereira, C. F., Nunes, E. F. C., \& Latorre, G. F. S. (2016). Função sexual feminina, desgaste emocional por insatisfação sexual e inteligência emocional. Fisioterapia Brasil, 17(6), 544-550.

Baigorria, J., Warmling, D., Magno Neves, C., Delziovo, C. R., \& Salema Coelho, E. B. (2017). Prevalência e fatores associados da violência sexual contra a mulher: revisão sistemática. Revista de Salud Pública, 19(6), 818-826. https://doi.org/10.15446/rsap.v19n6.65499

Barbosa, J. A. G., Souza, M. C. M. R., Souza, M. C. M. R., \& Freitas, M. I. F. (2015). Violência sexual: narrativas de mulheres com transtornos mentais no Brasil. Revista Panamericana de Salud Pública, 37(4/5), 2015.

Barreto, A. P. P., Nogueira, A., Teixeira, B., Brasil, C., Lemos, A., Lôrdelo, P. (2018). O impacto da disfunção sexual na qualidade de vida feminina: um estudo observacional. Revista Pesquisa em Fisioterapia, 8(4): 511-517.

Batista, N. M. T. L., Oliveira, A. N., Nunes, E. F. C., \& Latorre G. F. S. (2017). Força e coordenação motora da musculatura do assoalho pélvico e a função sexual feminina. Interdisciplinary Journal of Health Education, 2(1), 10-15. 10.4322/ijhe.2016.013

Bezerra, J. F., Silva, R. M., Cavalcanti, L. F., Nascimento, J. L., Vieira, L. J. E. S., \& Moreira, G. A. R. (2016). Conceitos, causas e repercussões da violência sexual contra a mulher na ótica de profissionais de saúde. Revista Brasileira em Promoção da Saúde, 29(1), 51-59. 10.5020/18061230.2016.p51

Campos, H. M., Nogueira, M. J., Fonseca, M. C., \& Schall, V. T. (2016). Saúde sexual, gênero e percepções de adolescentes sobre o preservativo feminino. Revista Adolescência e Saúde, 13(supl. 2), 26-32.

Cavalcante, A. E. O., Coutinho, G. B., Carvalho, A. R., Oliveira, E. T. de A., Silva, S. A., Moraes, L. M. V., Silva, F. M., \& Rodrigues, J. A. (2021). Mulheres profissionais do sexo: discurso sobre o uso do preservativo e sua autopercepção de vulnerabilidade ao HIV. Research, Society and Development, 10(2), e24010212440. 10.33448/rsd-v10i2.12440

Correa, S., Alves, J. E. D., \& Jannuzzi, P. M. (2015). Direitos e saúde sexual e reprodutiva: marco teórico-conceitual e sistema de indicadores. In: PATRIOTA, T. C. Indicadores municipais de saúde sexual e reprodutiva. Associação Brasileira de Estudos Populacionais (ABEP), cap. 1, p. $27-62$.

Cortes, H. M., Morais, A. V. C., Lacerda, L. C. S., Santos, R. O., \& Pinho, P. H. (2021). Sexualidade e Religiosidade: uma revisão integrativa de literatura. Research, Society and Development, 10(2), e37910212540. 10.33448/rsd-v10i2.12540

Cunha, L. M., Mota, W. S., Gomes, S. C., Ribeiro Filho, M. A., Bezerra I. M. P., Machado M. F. A. S., \& Quirino, G. S. (2015). Vovô e Vovó também amam: sexualidade na terceira idade. Revista Mineira de Enfermagem, 19(4), 894-906. 10.5935/1415-2762.20150069

Dantas, T. C., Silva, J. S. S., \& Carvalho, M. E. P. (2014). Entrelace entre gênero, sexualidade e deficiência: uma história feminina de rupturas e empoderamento. Revista Brasileira de Educação Especial, 20(4), 555-568. https://doi.org/10.1590/S1413-65382014000400007

Dantas-Berger, S. M., \& Giffin, K. (2005). A violência nas relações de conjugalidade: invisibilidade e banalização da violência sexual? Cadernos de Saúde Pública, 21(2), 417-425. https://doi.org/10.1590/S0102-311X2005000200008

Delziovo, C. R., Coelho, E. B. S., d'Orsi, E., \& Lindner, S. R. (2018). Violência sexual contra a mulher e o atendimento no setor saúde em Santa Catarina Brasil. Ciência \& Saúde Coletiva, 23(5), 1687-1696. https://doi.org/10.1590/1413-81232018235.20112016

Diamond, L. M., \& Alley, J. (2019). Was It Good for Her? An Alternative Perspective on Life History Theory, Female Same-Sex Sexuality, and Pleasure. Archives of sexual behavior, 48(5), 1315-1320. https://doi.org/10.1007/s10508-018-1313-5

Duffrayer, K. M., Mota, C. P., Silva, J. L. L., Messias, C. M., Moura, A. C. C. A. C., Silva, V. G. de F. E., Pereira, A. V., \& Silva, N. V. M. (2021). Perfil sociodemográfico de mulheres vítimas de violência no período de 2008 a 2017. Research, Society and Development, 10(4), e39710413823. 10.33448/rsdv10i4.13823

Fortes, D. C. S., Silva, M. R. S., Fonseca, K. S. G., \& Silva, A. S. B. (2021). Saúde sexual e reprodutiva da mulher com transtorno mental: fatores que moldam a assistência profissional. Research, Society and Development, 10(4), e49310414329. 10.33448/rsd-v10i4.14329 
Gaspar, R. S., \& Pereira, M. U. L. (2018). Evolução da notificação de violência sexual no Brasil de 2009 a 2013. Cadernos de Saúde Pública, 34(11), e00172617. https://dx.doi.org/10.1590/0102-311x00172617

Kingsberg, S. A., Schaffir, J., Faught, B. M., Pinkerton, J. V., Parish, S. J., Iglesia, C. B., Gudeman, J., Krop, J., \& Simon, J. A. (2019). Female Sexual Health: Barriers to Optimal Outcomes and a Roadmap for Improved Patient-Clinician Communications. Journal of women's health (2002), 28(4), 432-443. https://doi.org/10.1089/jwh.2018.7352

Lamont, J., Bajzak, K., Bouchard, C., Burnett, M., Byers, S., Cohen, T., Fisher, W., Holzapfel, S., \& Senikas, V. (2017). No. 279-Female Sexual Health Consensus Clinical Guidelines. Journal of obstetrics and gynaecology Canada: JOGC = Journal d'obstetrique et gynecologie du Canada: JOGC, 39(12), e535e541. https://doi.org/10.1016/j.jogc.2017.10.015

Mafioletti, T. M., Peres, A. M., Fontoura, M. P., Muzeka, K. D., Daltoé, C. M., \& Fellini, J. (2020). Rede de atenção às mulheres em situação de violência: experiência de um município do sul do Brasil. Research, Society and Development, 9(10), e1509108452. 10.33448/rsd-v9i10.8452

Mendonça, C. R., Arruda, J. T., Noll, M., Campoli, P., \& Amaral, W. (2017). Sexual dysfunction in infertile women: A systematic review and meta-analysis. European journal of obstetrics, gynecology, and reproductive biology, 215, 153-163. https://doi.org/10.1016/j.ejogrb.2017.06.013

Moraes, S. P., \& Vitalle, M. S. S. (2012). Direitos sexuais e reprodutivos na adolescência. Revista da Associação Médica Brasileira, 58(1), 48-52. https://doi.org/10.1590/S0104-42302012000100014

Oliveira, A. A. P., Villaça, L. A., Evangelista, M. G., Moreti, P. G., Brito, R. S., \& Ferreira, M. F. L. (2018). A relação entre saúde mental e sexual da mulher. Humanas \& Sociais Aplicadas, 8(22). 10.25242/887682220181540

Oliveira, C. A. B., Alencar, L. N., Cardena, R. R., Moreira, K. F. A., Pereira, P. P. S., \& Fernandes, D. E. R. (2019). Perfil da vítima e características da violência contra a mulher no estado de Rondônia-Brasil. Revista CUIDARTE, 10(1), e573. 10.15649/cuidarte.v10i1.573.

Pinto, L. S. S., Oliveira, I. M. P., Pinto, E. S. S., Leite, C. B. C., Melo, A. N., \& Deus, M. C. B. R. (2017). Políticas públicas de proteção à mulher: avaliação do atendimento em saúde de vítimas de violência sexual. Ciência \& Saúde Coletiva, 22(5), 1501-1508. https://dx.doi.org/10.1590/141381232017225.33272016

Ribeiro, J. N., \& Valle, P. A. S. S. (2016). Disfunção sexual feminina. Revista Brasileira de Sexualidade Humana, 27(2), 33-40. 10.35919/rbsh.v27i2.109

Rojas Cabrera, E., Moyano, S., \& Peláez, E. (2017). Progresos y desafíos en el ejercicio del derecho a la salud sexual y reproductiva en la Argentina de los últimos 40 años Una aproximación a partir de información relativa a "la primera vez". Sexualidad, Salud y Sociedad - Revista Latinoamericana, (25), $49-67$.

Scott, S. B., Ritchie, L., Knopp, K., Rhoades, G. K., \& Markman, H. J. (2018). Sexuality Within Female Same-Gender Couples: Definitions of Sex, Sexual Frequency Norms, and Factors Associated with Sexual Satisfaction. Archives of sexual behavior, 47(3), 681-692. https://doi.org/10.1007/s10508-017-1077-3

Sousa, M. M. M., \& Silva, N. C. (2018). Análise das ocorrências de violência sexual contra mulheres atendidas em um serviço de referência. Revista da Faculdade de Ciências Médicas de Sorocaba, 20(4), 195-199. 10.23925/1984-4840.2018v20i4a3

Sousa, M. H., Bento, S. F., Osis, M. J. D., Ribeiro, M. P., \& Faúndes, A. (2015). Preenchimento da notificação compulsória em serviços de saúde que atendem mulheres que sofrem violência sexual. Revista Brasileira de Epidemiologia, 18(1), 94-107. https://doi.org/10.1590/1980-5497201500010008

Ventriglio, A., \& Bhugra, D. (2019). Sexuality in the 21st Century: Sexual Fluidity. East Asian archives of psychiatry: official journal of the Hong Kong College of Psychiatrists = Dong Ya jing shen ke xue zhi : Xianggang jing shen ke yi xue yuan qi kan, 29(1), 30-34.

Viana, A. J. B., \& Sousa, E. S. S. (2014). O poder (in)visível da violência sexual: abordagens sociológicas de Pierre Bourdieu. Revista de Ciências Sociais, Fortaleza, 45(2), 155-183. 